\title{
Carcinoma oral de células escamosas e anos potenciais de vida perdidos: panorama no estado do Pará
}

\author{
Oral squamous cell carcinoma and the years of potential life lost: overview in the state of \\ Pará \\ El carcinoma oral de células escamosas y anos potenciales de vida perdidos: panorama \\ em el estado de Para
}

Pettra Blanco Lira Matos ${ }^{1 *}$, Lucas Carvalhaes Peres ${ }^{1}$, Andréa Cristina Marassi Lucas ${ }^{1}$, Bruno Vinícius dos Santos Matos ${ }^{2}$, Cecília Abrahão Nascimento de Santi ${ }^{1}$, Ana Maria Baia Cardoso ${ }^{1}$, Márcio Vinicius de Gouveia Affonso ${ }^{1}$, Helder Antonio Rebelo Pontes ${ }^{1}$, Flávia Sirotheau Corrêa Pontes $^{1}$, Liliane Silva do Nascimento ${ }^{1}$.

\section{RESUMO}

Objetivo: Avaliar o índice de Anos Potenciais Perdidos por pessoas diagnosticadas com Carcinoma oral de células escamosas no Estado do Pará. Métodos: Estudo observacional e ecológico. Realizou-se uma análise da distribuição do Carcinoma oral de células escamosas no Estado do Pará com dados obtidos de 318 pacientes acometidos por este carcinoma e atendidos em um hospital de referência, no período de janeiro de 2007 a agosto de 2016. Calculou-se o índice de anos potenciais de vida perdidos pela fórmula adaptada de Romeder e McWhinie. Realizou-se consulta por meio do Termo de cessão de dados (TCD). Resultados: A idade de maior acometimento é na sexta década de vida, onde os homens se sobressaem em ralação às mulheres. Cerca de $61 \%$ dos pacientes eram tabagistas ao diagnóstico e aproximadamente $44,65 \%$ não faziam uso de bebidas alcoólicas. A língua se mostrou como sítio de maior acometimento das lesões. Foi calculado 921,5 anos de vidas perdidas para os pacientes estudados. Conclusão: Estes estudos servem como base para verificar impactos econômicos e sociais que o carcinoma carrega, ajudando a direcionar políticas públicas para o diagnóstico precoce e uma melhor capacitação dos profissionais que trabalham nesta área, podendo evitar gastos dispendiosos para a saúde no futuro.

Palavras-chave: Carcinoma de células escamosas, Anos potenciais de vida perdidos, Saúde pública.

\section{ABSTRACT}

Objective: To evaluate the index of Potential Years Lost by people diagnosed with oral squamous cell carcinoma in the State of Pará. Methods: Observational and ecological study. An analysis of the distribution of oral squamous cell carcinoma in the State of Pará was carried out with data obtained from 318 patients affected by this carcinoma and treated at a referral hospital, from January 2007 to August 2016. The Index of potential years of life lost by the formula adapted from Romeder and McWhinie. Consultation was carried out using the Data Assignment Term (TCD). Results: The most affected age is in the sixth decade of life, where men stand out in relation to women. About $61 \%$ of the patients were smokers at diagnosis and approximately $44.65 \%$ did not use alcohol. The language is English as the most affected site for children. It was a total of 921.5 years of lives lost for the patients studied. Conclusion: These studies serve as a basis for correcting the impacts and social impacts of carcinoma, helping to direct public policies for early diagnosis and better training for professionals working in this area, which can avoid costly expenses for health in the future.

Key words: Squamous cell carcinoma, Potential years of life lost, Public health.

\section{RESUMEN}

Objetivo: Evaluar el índice de Años Potenciales Perdidos por personas diagnosticadas con carcinoma oral de células escamosas en el Estado de Pará. Métodos: Estudio observacional y ecológico. Se realizó un análisis de la distribución del carcinoma oral de células escamosas en el estado de Pará con datos obtenidos de 318 pacientes afectados por este carcinoma y atendidos en un hospital de referencia, desde enero de 2007 hasta agosto de 2016. El Índice de años potenciales de vida perdidos por la fórmula adaptada de Romeder y

1 Universidade Federal do Pará (UFPA), Belém - PA. * E-mail: pettra_lira@yahoo.com.br

${ }^{2}$ Centro Universitário Augusto Motta (UNISUAM), Rio de Janeiro - RJ.

SUBMETIDO EM: 6/2020

ACEITO EM: 7/2020

PUBLICADO EM: 9/2020

REAS/EJCH | Vol.Sup.n.57 | e4092 | DOI: https://doi.org/10.25248/reas.e4092.2020 Página 1 de 8 
McWhinie. La consulta se llevó a cabo mediante el término de asignación de datos (TCD). Resultados: La edad más afectada se encuentra en la sexta década de la vida, donde destacan los hombres en relación a las mujeres. Aproximadamente el $61 \%$ de los pacientes eran fumadores en el momento del diagnóstico y aproximadamente el 44,65\% no consumía alcohol. El idioma es el inglés como el sitio más afectado por los niños. Fue un total de 921,5 años de vidas perdidas para los pacientes estudiados. Conclusión: Estos estudios sirven de base para corregir los impactos e impactos sociales del carcinoma, ayudando a orientar las políticas públicas para el diagnóstico temprano y una mejor capacitación de los profesionales que trabajan en esta área, lo que puede evitar costosos gastos para la salud en el futuro.

Palabras clave: Carcinoma de células escamosas, Posibles años de vida perdidos, Salud pública.

\section{INTRODUÇÃO}

O estudo da população ao longo dos tempos revela que, nos dias atuais, vive-se um processo chamado de "envelhecimento da população", onde se faz presente a chamada transição epidemiológica, que leva a uma transformação específica: a diminuição da ocorrência de doenças infectocontagiosas e o aumento progressivo das doenças crônico-degenerativas. Este último grupo de doenças se encontra em evidência devido ao aumento progressivo da morbimortalidade, o que tem ampliado a vigilância das mesmas, e se faz importante na produção de dados que auxiliem a tomada de decisões (WHO, 2002).

Dentre as doenças crônico-degenerativas se inclui o câncer, o qual é caracterizado por um crescimento desordenado das células em uma determinada região. O subtipo que atinge a cavidade oral é denominado de "câncer oral", e abrange de acordo com a Classificação Internacional de Doenças (CID) as determinadas regiões anatômicas: lábio, base da língua, partes não especificadas da Língua, gengiva, assoalho da Boca, palato, partes não especificadas da boca, glândula parótida, glândulas salivares maiores e não especificadas, amígdala e orofaringe (WHO, 2002; FREITAS R, et al., 2016).

No Brasil, são registrados cerca de 390 mil casos de câncer de boca e orofaringe anualmente (ARAUJO ACS, et al., 2019). Dentre esses, o Carcinoma de células escamosas (CCE) é a neoplasia maligna mais comumente encontrada na região oral e apresenta uma origem multifatorial, com fatores predisponentes tanto extrínsecos como o álcool, radiação, tabaco e outros; quanto intrínsecos como a herança genética e predisposição familiar (FREITAS R, et al., 2016; VALLE CN, et al., 2016; PETITO G, et al., 2017; ARAUJO ACS, et al., 2019).

Esta neoplasia se caracteriza como uma lesão agressiva, a qual geralmente é diagnosticada em estágio avançado, mesmo apresentando sinais clínicos desde o seu início; porém, frequentemente assintomática nesta fase, o que dificulta o diagnóstico precoce. Dessa forma, políticas públicas voltadas para este objetivo se apresentam de fundamental importância no planejamento de ações de prevenção e controle do câncer (SANTOS LCO, et al., 2010; VALLE CN, et al., 2016; PETITO G, et al., 2017).

Estudar uma determinada doença a partir do indicador de mortalidade é relevante, porém, outros fatores são considerados quando se faz uso de outro indicador, neste caso os Anos Potenciais de Vida Perdidos (APVP). Um exemplo a ser demonstrado foi o estudo realizado pelo próprio criador deste indicador, Dempsey, que comparou as mortes causadas por tuberculose com as causadas por doenças cardiovasculares e por câncer. Os resultados do estudo foram de extrema importância, pois a gravidade da tuberculose foi redimensionada, o que em outro momento, era subestimada nas análises que se basearam apenas nas taxas de mortalidade (MEDRONHO RA, et al., 2009).

Alguns indicadores utilizados atualmente para o estudo de determinadas doenças que acometem a população brasileira são insuficientes ou incapazes de representar a devida importância da doença na sociedade. As taxas de mortalidades são insuficientes para atribuir um determinado valor para a sociedade quando falamos de mortes causadas por doenças em uma dada população, pois se limitam a descrever apenas a quantificação das mesmas (REICHENHEIM ME e WERNECK GL, 1994).

O indicador APVP se torna uma boa opção no sentido de expressar o valor social, visto que se trata de uma medida de mortalidade que não enfatiza apenas a frequência com que os óbitos ocorrem; no entanto, vai além, dimensiona o tempo que uma pessoa deixou de viver no momento de sua morte, usando como 
referência a expectativa de vida de uma determinada população. Assim, além de se basear na quantidade de óbitos, os APVP se sustentam no critério de transcendência e expressão social atribuído ao problema em questão (MEDRONHO RA, et al., 2009).

Dessa forma, esse indicador se fundamenta no princípio de que cada pessoa tem o potencial de viver até um determinado número de anos, caso este morra com antecedência, em uma etapa de vida considerada potencial de alta produtividade, se traduz em perdas importantes para a sociedade, devido à privação de sua provável contribuição, de forma intelectual ou na economia (WU CHEN E, et al., 2019). Nesse sentido, o objetivo deste estudo é avaliar o índice de Anos Potenciais de Vida Perdidos por indivíduos que foram diagnosticados com Carcinoma Oral de Células Escamosas no Estado do Pará, estimulando o redirecionamento na gestão das políticas públicas.

\section{MÉTODOS}

Trata-se de estudo observacional e ecológico, cuja amostra foi retirada do banco de dados de pacientes acometidos por Carcinoma de células escamosas da cavidade oral atendidos pelo setor de Patologia Bucal de um hospital universitário localizado na cidade de Belém, Pará (Brasil), no período de Janeiro de 2007 a Agosto de 2016. Foi realizada consulta através do Termo de Cessão de Dados (TCD), assinado pelo responsável da tabela de pacientes. Todas as etapas seguiram as determinações da pesquisa com seres humanos sob o parecer do CEP 2.481.506.

De um total de 318 casos de pacientes diagnosticados no referido período, 113 vieram a óbito, dos quais 66 entraram no cálculo de APVP pois apresentavam idade de óbito que se encontrava dentro dos limites propostos pela fórmula pré-estabelecida. Na referida tabela, continham informações relativas ao sexo, idade, localização da lesão, estadiamento, local de moradia, idade do óbito, sobrevida e realização de quimioterapia e radioterapia.

Após análise e coleta de dados, estes foram tabulados descrevendo como o Carcinoma de células escamosas oral ocorre no Estado do Pará, mostrando a prevalência nos sexos, a frequência de pacientes fumantes e os que faziam uso de bebidas alcoólicas, grau de estadiamento no momento do diagnóstico e locais de maior prevalência de acometimento na cavidade oral.

Em seguida foram realizados os cálculos dos Anos Potenciais de Vida Perdidos (APVP), seguindo a fórmula desenvolvida por Romeder JM e McWhinnie JR (1977), amplamente utilizada nestes estudos, que usa como limite inferior a idade de 01 ano e como limite superior a idade de 70 anos.

Os óbitos infantis foram excluídos pelo fato da maioria estar relacionada a causas especificas deste primeiro ano de vida. Óbitos por causas mal definidas em idades mais avançadas, justificam o limite superior de 70 anos (MEDRONHO RA, et al., 2009).

Basicamente, se faz a multiplicação do número de óbitos que ocorreram em determinada idade pelo número de anos que faltam para atingir a idade limite pré-determinada pela fórmula, no caso 70 anos. $\mathrm{O}$ cálculo é realizado para cada idade de óbito dos pacientes de maneira individual. Em seguida, se realiza a soma dos resultados para se obter o total de APVP. Desse modo, os óbitos que ocorrem em idades inferiores somam um número maior de Anos potenciais perdidos quando comparados com a de idades superiores, próximas aos 70 anos. O cálculo da média de Anos Potenciais Perdidos foi realizado através da divisão do total de APVP encontrado pelo número de óbitos que foi utilizado na fórmula geral, obtendo assim a média de Anos perdidos por cada paciente.

\section{RESULTADOS}

Diante dos dados obtidos pôde ser observado os intervalos de idades referentes aos diagnósticos dos 318 pacientes acometidos pelo Carcinoma Oral de Células Escamosas, especificando o gênero dos mesmos (Gráfico 1). Foi aferido um maior número de diagnósticos na sexta década de vida e uma predominância do sexo masculino. Os únicos períodos em que os diagnósticos do sexo feminino ultrapassaram os masculinos são observados na sétima e nona décadas de vida. As informações a respeito das idades de 14 pacientes nãos constavam no gráfico. 
Gráfico 1 - Número de casos de Carcinomas de células escamosas da cavidade oral (bruto) referentes aos intervalos de idades, utilizando o sexo como variável.

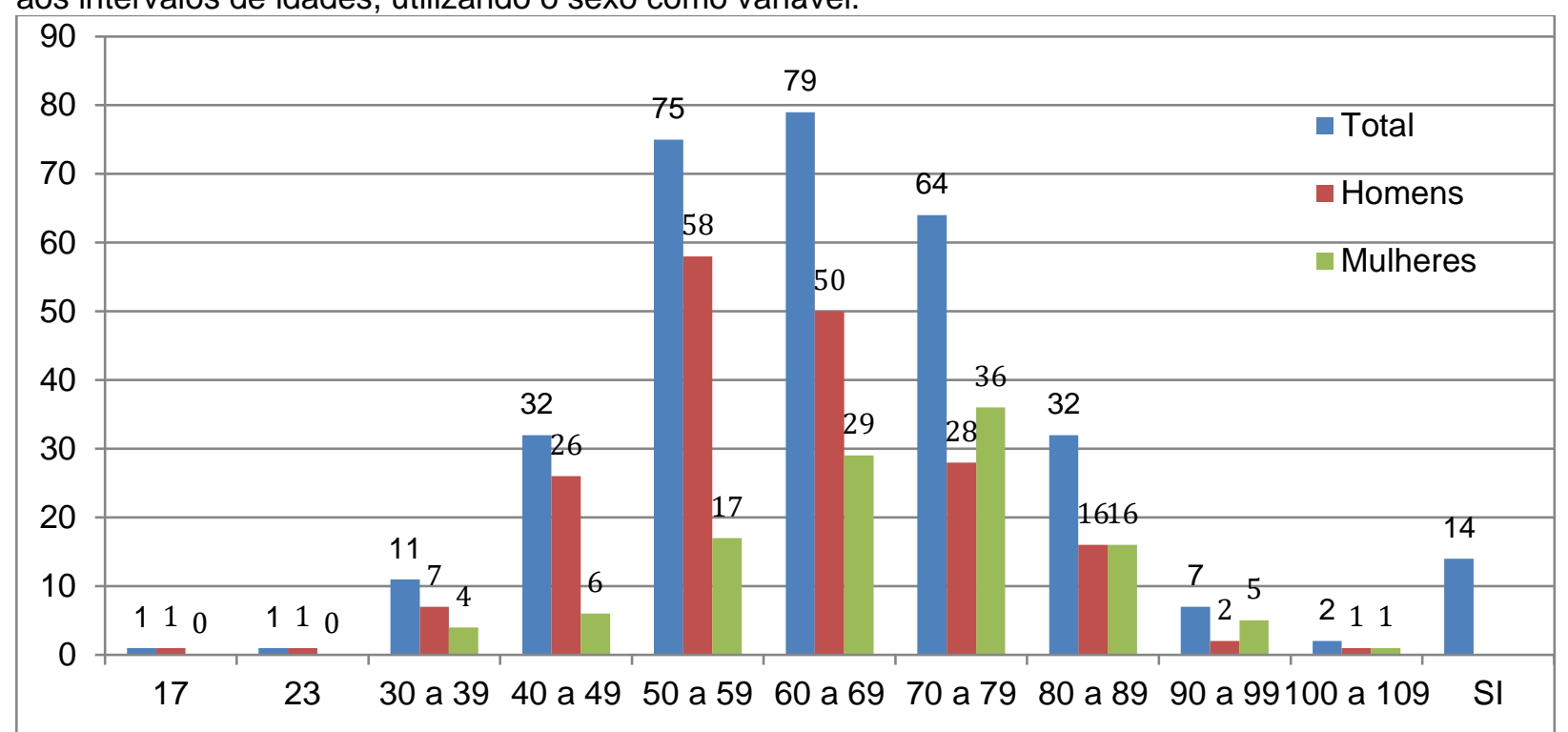

Fonte: Matos PBL, et al., 2020.

A amostra deste estudo foi composta por 318 diagnósticos; destes 198 foram atribuídos ao sexo masculino e 120 ao sexo feminino. Em relação ao hábito de fumar, 97 pacientes relataram não ser tabagistas, enquanto 195 relataram apresentar este hábito. Do total dos casos, 26 não constavam tal informação e apenas 01 indivíduo afirmou já ter feito uso de cigarro em algum momento da vida. Quanto ao consumo de bebida alcoólica, 136 afirmaram exercer esta prática e 142 relataram que não; enquanto que 40 casos não constavam tal informação. Em relação à localização e extensão da lesão, a grande maioria dos casos do carcinoma foram encontrados na língua e extensões para a língua (Tabela 1).

Tabela 1 - Número de pessoas diagnosticadas com Carcinoma de células escamosas da cavidade oral (bruto e percentagem), relacionados com as variáveis: sexo, tabagismo, etilismo, e extensão da lesão.

\begin{tabular}{cccc}
\hline & Descrição & $\mathbf{n}$ & $\%$ \\
\hline \multirow{2}{*}{ Sexo } & Feminino & 120 & $38 \%$ \\
& Masculino & 198 & $62 \%$ \\
\hline \multirow{2}{*}{ Tabagismo } & Não & 97 & $30,50 \%$ \\
& Sim & 195 & $61,32 \%$ \\
\hline \multirow{2}{*}{ Etilismo } & Não & 142 & $44,65 \%$ \\
& Sim & 136 & $42,76 \%$ \\
\hline & Língua/ extensões para língua & 88 & $28,8 \%$ \\
& Soalho/ extensões para & & \\
& Soalho & 23 & $7,5 \%$ \\
Localização/ & Região maxilar/ extensões & & \\
& para maxila & 27 & $8,8 \%$ \\
& Região mandibular/ extensões & & \\
& para a mandíbula & 21 & $6,8 \%$ \\
& Palato duro e extensões & 27 & $7,5 \%$ \\
& Palato mole e Orofaringe e & & $15,4 \%$ \\
& extensões & 47 & $4,9 \%$ \\
& Lábio inferior & 15 & $1,3 \%$ \\
& Lábio Superior & 4 & $13,1 \%$ \\
& Mucosa jugal & 40 & $4,2 \%$ \\
& Outros & 13 &
\end{tabular}

Fonte: Matos PBL, et al., 2020. 
À frente dos resultados obtidos em relação ao estadiamento dos tumores, pôde ser concluído que a maioria deles pertencia ao estágio III no momento do diagnóstico; segundo o sistema TNM de estadiamento (Tabela 2).

Tabela 2 - Porcentagem de pacientes em relação ao estágio do tumor no momento do diagnóstico, segundo o sistema TNM de estadiamento.

\begin{tabular}{ccc}
\hline Estágio do tumor & № pacientes (\%) \\
\hline I & $13,9 \%$ \\
II & & $20,3 \%$ \\
III & IIVA & $26,6 \%$ \\
& IV & $20,3 \%$ \\
& IIVB & $3,3 \%$ \\
& IIVC & $0 \%$ \\
\hline
\end{tabular}

Fonte: Matos PBL, et al., 2020.

Com a tabulação dos dados referentes aos diagnósticos dos 318 pacientes acometidos por Carcinoma de células escamosas da cavidade oral no Hospital Universitário, foram calculados os Anos potenciais de vida perdidos para os 113 pacientes que vieram a óbito, onde se obteve um valor total de 921,5 anos de vida perdidas através da fórmula de Romeder JM e McWhinnie JR (1977), já previamente estabelecida, no período de Janeiro de 2007 a Agosto de 2016.

Com referência aos 921,5 anos de vida; 739 anos pertenciam ao sexo masculino e 182,5 anos ao sexo feminino. Assim como a média de APVP calculada para cada pessoa foi de 14,1 anos. Com relação à distribuição dos óbitos ocorridos, se constatou que $63,7 \%$ eram do sexo masculino, $70 \%$ eram tabagistas e $51,3 \%$ faziam uso de bebidas alcoólicas (Tabela 3 ).

Tabela 3 - Número de óbitos por pessoas diagnosticadas com Carcinoma de células escamosas da cavidade oral (bruto e percentagem), relacionados com as variáveis: sexo, tabagismo, etilismo.

\begin{tabular}{cccc}
\hline & Descrição & $\mathbf{n}$ & $\%$ \\
\hline \multirow{2}{*}{ Sexo } & Feminino & 41 & $36,3 \%$ \\
& Masculino & 72 & $63,7 \%$ \\
\hline \multirow{2}{*}{ Tabagismo } & Não & 34 & $30,0 \%$ \\
& Sim & 79 & $70,0 \%$ \\
\hline \multirow{2}{*}{ Etilismo } & Não & 55 & $48,7 \%$ \\
& Sim & 58 & $51,3 \%$ \\
\hline
\end{tabular}

Fonte: Matos PBL, et al., 2020.

\section{DISCUSSÃO}

O Carcinoma Oral de Células Escamosas compõe uma das neoplasias malignas mais comumente diagnosticadas, apresentando mais de $50 \%$ de pacientes em estágio avançado. Quanto à caracterização da amostra, há um maior número de casos diagnosticados no sexo masculino $(62 \%)$ e a prevalência de idade na sexta década de vida; o que se assemelha com outros achados na literatura, sendo esta relação, provavelmente, em decorrência a um maior tempo de exposição destes indivíduos aos fatores de risco (SILVA LAB, 2016; VALLE CN, et al., 2016; RAMOS TCF, et al., 2019).

O número de casos diagnosticados do sexo feminino foi de $38 \%$, representando um pouco mais de um terço da amostra; fato este que expressa a mudança nos hábitos de vida das mulheres, que passaram a apresentar exposição mais frequente aos agentes carcinógenos. Tem sido observado que essa lesão está cada vez mais visível nas mulheres por estarem fumando e fazendo uso de bebidas alcoólicas no mesmo nível que os homens (DOMINGOS, PASSALACQUA E OLIVEIRA, 2014; EMERICK C, et al., 2020).

Diante dos resultados, ao se levar em consideração a faixa etária da amostra, apesar dos idosos serem o grupo etário mais acometido pela doença; tem sido observado um aumento do número de casos diagnosticados em pacientes mais jovens nos últimos anos. Nesta pesquisa, $11(3,46 \%)$ pacientes pertenciam 
ao grupo da terceira década de vida, assim como 32 (10,06\%) pacientes pertenciam a quarta década de vida. A observação desses dados demonstra que os indivíduos estão sendo expostos aos fatores de riscos mais associados ao desenvolvimento deste tipo de neoplasia mais precocemente, conforme pesquisas já realizadas (SILVA LAB, 2016; RAMOS TCF, et al., 2019; EMERICK C, et al., 2020).

Foi observado um número de 113 mortes de pacientes no período de tempo estudado, o que corresponde a $35,5 \%$ do valor total de pacientes diagnosticados. Destes $63,7 \%$ eram do sexo masculino, corroborando com vários estudos presentes na literatura (FERNÁNDEZ A, et al., 2015; RAMIREZ V, et al., 2015; ROVITO MJ, 2016).

Assim como foi observado que os $70 \%$ fumante e $51,3 \%$ que consumiam bebidas alcóolicas estão em concordância a outros achados na literatura, onde a maioria dos pacientes diagnosticados eram fumantes e também grande parte deles consumiam bebidas alcoólicas; demostrando que o tabagismo e etilismo são fatores que influenciam no desenvolvimento do carcinoma (LEMOS-JÚNIOR CA e VILLORIA GEM, 2008; SANTOS GL; 2010; SOUSA FB, 2014; VOLKWEIS MR, et al., 2014; ARAÚJO ACS, et al., 2019)

Algumas pesquisas realizadas corroboram com este estudo ao demonstrar que a localização mais frequente de acometimento do carcinoma de células escamosas na região oral foi a língua e extensões para a língua, o que reflete uma informação importante aos cirurgiões-dentistas para exame clinico com atenção minuciosa a lesões nessa localização anatômica e em especial aos pacientes que apresentem maior exposição aos fatores de risco, em virtude dessa região apresentar maior risco de envolvimento linfonodal (SANTANA ITS, 2018; RAMOS TCF, et al., 2019; EMERICK C, et al., 2020).

Outra semelhança encontrada com alguns estudos já realizados foi o estadiamento dos tumores. $O$ estágio do tumor mais comumente encontrado no momento do diagnóstico foi o III com $26 \%$ dos casos, considerado um estágio avançado; assim é possível verificar a falta de atenção no que diz respeito a busca por cuidados em saúde e com isso tornando o prognóstico mais sombrio (MAMERI HÁ, 2015; ASSIS ACMG, 2018). Há inúmeros fatores que tornam mais complexos o diagnóstico precoce das lesões, como a ausência de sintomas da doença em sua fase inicial e a falta de profissionais capacitados para estabelecer um diagnóstico precoce (DOMINGOS, PASSALACQUA E OLIVEIRA, 2014).

O indicador Anos Potenciais de Vidas Perdidos em conjunto com a exposição das características dos pacientes acometidos por carcinoma de células escamosas da cavidade oral demonstrados, se torna uma importante ferramenta para o futuro planejamento e execução de políticas públicas, enfatizando o diagnóstico precoce do Carcinoma, principalmente no âmbito da Atenção primária em saúde, que pode ser realizado através de exames simples de rotina e anamnese detalhada; ações importantes para minimizar os riscos de vida para o paciente. Esta necessidade é reforçada frente ao resultado acerca do estágio avançado do tumor, casos em que os tumores são maiores, e que neste estágio já pode apresentar metástases, o que dificulta o seu tratamento e sua remoção, e ainda facilitando possíveis recidivas loco-regionais ou à distância (JOHN U e HANKE M, 2015).

O diagnóstico nesse estágio ocasiona taxas de morbidade e mortalidade elevadas com sobrevida de até 05 anos, sendo dependente das particularidades de cada indivíduo como características socioeconômicas, raça, educação preventiva, expectativa de vida e qualidade de assistência medica (VALLE CN, et al., 2016; ARAUJO ACS, et al., 2019). Dessa forma é de extrema relevância estimar os custos das mortes que uma determinada doença traz para a sociedade, e nesse contexto o indicador APVP consegue desempenhar bem este papel ao agregar valor social aos óbitos (JOHN U e HANKE M, 2015).

É válido e muito importante em determinados casos ser aplicado índices que permita essa visão mais social de uma determinada doença, fugindo um pouco da individualidade da mesma e contrariando sua quantificação isolada, como por exemplo, relativizar os óbitos de uma doença com a carga econômica que ela impactará. A perda de anos potenciais de vida pode estar relacionando com o tempo de trabalho perdido e o ano de aposentadoria, expondo diversos fatores aos riscos, principalmente os econômicos, como perda de salários recebidos, arrecadações perdidas e custos com o tratamento da doença (ALMEIDA MEL, et al., 2002; ROVITO MJ, 2016; WU CHEN E, et al., 2019). 
Na literatura é encontrado o chamado estudo de custo de doença, no qual é exibido os custos diretos (hospitalares, médicos, visitas, transporte, comidas, etc) e os indiretos (não ir ao trabalho, perda de produtividade perante o óbito e aposentadoria antecipada). Neste sentido, se destaca a importância de quantificar o valor da perda de trabalho e os custos da doença para direcionar gastos públicos e também para a criação de políticas públicas (NAJAFI F, et al., 2016). Logo, o estudo direcionado para os APVPs de uma determinada doença é de suma importância para redimensionar o impacto que esta traz para a sociedade no que diz respeito à economia e à criação de políticas públicas (GAY GP e EKWUEME DU, 2011; JOHN U e HANKE M, 2015; KRISTINA SA, et al., 2015; KARAMI-MATIN B, et al., 2016).

É possível observar que a diferença é bem considerável quando comparamos os Anos potenciais de vida perdidos entre Janeiro de 2007 e Agosto de 2016 pelo sexo masculino, 739 anos, com o sexo feminino, 182,5. É provável que isto ocorra pelo fato de que no estudo foi observado mais óbitos do sexo masculino do que do sexo feminino. A média de APVP obtida para as variáveis foi de 15,3 anos no sexo masculino e 10,7 anos para o sexo feminino. Os valores de APVP por óbitos obtidos para tais variáveis, se assemelham aos resultados presentes na literatura, na qual também se observa que a média de APVP em homens é mais alta do que em mulheres, no que se refere ao Câncer da cavidade oral (ALMEIDA MEL, et al., 2002; PEREA LME, et al., 2019).

A realização de estudos analisando o comportamento das doenças crônicas é crucial para posterior desenvolvimento de estratégias de prevenção. As altas taxas de anos de vida perdidos por câncer, assim como por outras doenças crônicas, se traduzem em uma grande sobrecarga nos sistemas de saúde e impacto econômico para as famílias e para a sociedade. Dessa forma, esses fatores podem levar ao prejuízo social e empobrecimento (PEREA LME, et al., 2019).

Se faz Importante ressaltar algumas limitações do estudo, como a falta de preenchimento de algumas informações do banco de dados, o que pode afetar valores das variáveis "Etilismo", "Localização da lesão" e "Estadiamento". Foi observado perda de segmento de alguns pacientes também. Porém, para o autor, isto não influenciou de maneira expressiva, já que eram poucos casos que se enquadravam em tais deficiências de informações. Outro fator importante, que no caso interferiu nos valores do índice APVP, foi 0 estabelecimento de 70 anos como limite superior pré-estabelecido pela fórmula utilizada de Romeder JM e McWhinnie JR (1977) o que exclui um número considerável de óbitos que poderiam influenciar o valor final do índice. Ou seja, apenas os óbitos que ocorreram em idades abaixo de 70 anos compunham a fórmula e consequentemente, o valor de anos potenciais perdidos.

\section{CONCLUSÃO}

O Carcinoma de células escamosas da cavidade oral se trata de uma das neoplasias mais comuns, que leva à morbidade e mortalidade, mostrando assim, quão arrasadoramente esta doença afeta os fatores econômicos e sociais. A análise situacional do Carcinoma nesta pesquisa se mostrou semelhante ao descrito na literatura, sendo este agravo uma questão impactante na saúde pública e que se diagnosticado precocemente possui maior chance de tratamento e pode evitar gastos dispendiosos com a saúde. A diferença obtida no padrão de mortalidade entre os sexos, medidas a partir do índice APVP, é bastante relevante e uma investigação epidemiológica a partir deste indicador a respeito das mortalidades de homens acometidos por carcinoma de células escamosas oral se faz necessário.

\section{AGRADECIMENTOS E FINANCIAMENTO}

Agradecemos ao Hospital Universitário da Universidade Federal do Pará pelo apoio na realização deste estudo.

\section{REFERÊNCIAS}

1. ALMEIDA MEL, et al. Potencial Years of Life Lost and Mortality of Mouth and Pharynx Cancer. RGO - Revista Gaúcha de odontologia. 2002; 50(1): $21-24$.

2. ARAÚJO ACS, et al. Ocorrência sincrônica de Carcinoma Espinocelular Bucal Microinvasor e Adenocarcinoma Pulmonar: relato de caso. REAS/EJCH. 2019; 11(12): 1-9. 
3. ASSIS ACM. Estudo retrospectivo do carcinoma de células escamosas bucal diagnosticado em um centro de pesquisa. 2018.30f. Trabalho de conclusão de curso em odontologia, Uberaba, 2018.

4. DOMINGOS PAS, PASSALACQUA MLC, OLIVEIRA ALBM. Câncer bucal: um problema de saúde pública. Ver Odontol Univ Cid São Paulo, 2014; 26(1): 46-52.

5. EMERICK C, et al. Perfil sociodemografico e clinicopatológico de 80 casos de Carcinoma de células escamosas de boca. J Bras Patol Med LAb, 2020; 56: 1-6.

6. FERNÁNDEZ A, et al. Epidemiological characterization of oral cancer. Literature review. Journal of oral research. 2015; 4(2): 137-145.

7. FREITAS R, et al. Risk factors and major cytopathologycal changes of oral cancer: a review of literature. Revista Brasileira de Análises Clínicas, 2016; 48(1): 13-18.

8. GAY GP, EKWUEME DU. Years of Potential Life Lost and Indirect Costs of Melanoma and Non-Melanoma Skin Cancer. A Systematic Review of the Literature. Pharmacoeconomics. 2011; 29(10): 863 - 874.

9. JOHN U, HANKE M. Lung cancer mortality and years of potential life lost among males and females over six decades in a country with high smoking prevalence: an observational study. BMC Cancer. 2015; 876(15): 1-9.

10. KARAMI-MATIN B, et al. Estimating the Economic Burden of Premature Mortality Caused by Cancer in Iran: 2006-2010. Asian Pac J Cancer Prev. 2016; 17(4): 2131-2136.

11. KRISTINA SA, et al. Burden of Cancers Related to Smoking among the Indonesian Population: Premature Mortality Costs and Years of Potential Life Lost. Asian Pacific Journal of Cancer Prevention. 2015; 16(16): 6903-6908.

12. LEMOS-JÚNIOR CA, VILLORIA GEM. Reviewed evidence about the safety of the daily use of alcohol-based mouthrinses. Braz. oral res. 2008; 22(1): 24-31.

13. MAMERI HA. Relação entre tabagismo e o etilismo e o carcinoma de células escamosas na região de cabeça e pescoço em pacientes usuários do SUS no Espirito Santo. 2015. 86f. Dissertação (Mestrado em clinica odontológica). Universidade Federal do Espirito Santo, Vitoria, 2015.

14. MEDRONHO RA, BLOCH KV, LUIZ RR, WERNECK GL. Epidemiologia. 2nd ed. Rio de Janeiro: Atheneu, 2009; 676p.

15. NAJAFI F, et al. Productivity costs and years of potential life lost associated with five leading causes of death: Evidence from Iran (2006-2010). Med J Islam Repub Iran. 2016; 412(30): 1-8.

16. PEREA LME, et al. Anos potenciais de vida perdidos por câncer de boca e faringe no Brasil:1979 a 2013. Revista de saúde publica, 2019; 53(67): 1-11.

17. PETITO G, et al. Human papillomavirus in oral cavity and oropharynx carcinomas in the central region of Brazil. Braz J Otorhinolaryngol, 2017; 83: 38-44.

18. RAMIREZ V, et al. Oral and pharyngeal cancer mortality in Chile, years 2002-2010. Revista Clínica de Periodoncia, Implantología y Rehabilitación Oral. 2015; 8(2): 133-138.

19. RAMOS TCF, et al. Perfil clinico-demográfico de los carcinomas de células escamosas bucales em uma poblacion del nordeste de Brasil. Ver Asoc Odontol Argent, 2019; 107:5-9.

20. REICHENHEIM ME, WERNECK GL. Anos Potenciais de Vida Perdidos no Rio de Janeiro, 1990. As Mortes Violentas em Questão. Cad. Saúde Públ., Rio de Janeiro, 1994; 10 (supl. 1): 188-198.

21. ROMEDER JM, McWHINNIE JR. Potential years of life lost between ages 1 and 70: an indicator of premature mortality for health planning. Int J Epidemiol. 1977; 6(2): 143-51.

22. ROVITO MJ. Eclipsed by the Prostate: Expanding Testicular Cancer Scholarship Through Years of Potential Life Lost and Economic Productivity. American Journal of Men's Health. 2016; 11(3): 1-4.

23. SANTANA ITS. Valor prognóstico dos polimorfismos funcionais nos genes da pon1, tnf- $\alpha$ e tgf- $\beta$ no carcinoma de células escamosas oral e orofaríngeo. 2018. Dissertação (Mestrado em ciências aplicadas a saúde). Universidade Federal de Sergipe, Lagarto, 2018.

24. SANTOS GL, et al. Tobacco and alcohol as risk factors for buccal cancer. Odontol Clín-Cient. 2010; 9(2): 131-133.

25. SANTOS LCO, et al. Caracterização do diagnóstico tardio do câncer de boca no estado de Alagoas. Braz. j. otorhinolaryngol, 2010; 76(4): 416-22.

26. SILVA LAB. Correlação da imunossupressão do fator de choque térmico 1 (HSF1) com aspectos clinicopatológicos em Carcinoma de Celulas Escamosas de língua oral. 2016. 125f. Dissertação (Mestrado em Patologia Oral). Universidade Federal do Rio Grande do Norte, Natal, 2016.

27. SOUSA FB, et al. Oral cancer from a health promotion perspective: experience of a diagnosis network in Ceará. Braz Oral Res., 2014; 28(Spec Iss 1): 1-8.

28. VALLE CN, et al. Carcinoma espinocelular oral: um panorama atual. Ver Pato Tocantins, 2016; 4(3): 82-102.

29. VOLKWEIS MR, et al. Epidemiologic Profile of the Patients with Oral Cancer in a CEO. Rev Cir Traumatol Buco-MaxiloFac. 2014; 14(2): 63-70.

30. WORLD HEATH ORGANIZATION (WHO). Policies and managerial guidelines for national cancer control programs. Rev Panam Salud Publ, 2002; 12(5): 366-70.

31. WU CHEN E, et al. Anos potenciais de vida perdidos no período de 2010 a 2015 em Foz do Iguaçu, Paraná, Brasil. Rev. Bras. De Iniciação Científica (RBIC). 2019; 6(4): 18-33. 